\title{
SUMMARY REPORT OF THE SPIN PHYSICS WORKING GROUP *
}

\author{
NICOLA BIANCHI \\ INFN - Laboratori Nazionali di Frascati, \\ via E. Fermi 40, I-00044 Frascati, Italy \\ E-mail: bianchi@hermes.desy.de \\ RAINER JAKOB \\ Fachbereich Physik, Universität Wuppertal, \\ 42097 Wuppertal, Germany \\ E-mail: rainer@theorie.physik.uni-wuppertal.de
}

\begin{abstract}
The contributions to the Spin Physics WG are summarized. Several new experimental results and plans for new measurements have been reported. An improved theoretical understanding of the most recent hot topics in spin physics has been discussed by many authors especially in the new fields of the transversity and generalized parton distributions.
\end{abstract}

\section{DIS $_{2001 \text {-claimer }}$}

The spin physics working group at the DIS 2001 workshop hosted a total of 37 talks equally distributed between experimental and theoretical reports. The topics covered many different aspects of polarization phenomena in deep inelastic scattering. This summary presents recent developments reported at the workshop, and sketches the contours of the ongoing world wide spin program in hadron physics. Unavoidably, a summary cannot reflect all facets and details of such a variety of contributions in a fair manner. Sorry!

\section{Introduction}

Spin - merely an inessential complication in particle physics ? No, anything but that! On the contrary, over and over again spin physics proves to be a vital element and indispensable tool in our venture of revealing the internal structure of hadronic matter. Once again, at this workshop, the numerous contributions, above average attendance, and lively discussions in the parallel sessions of the spin physics working group testified the vividness of the field. The physics topics were divided in five different subsections, namely:

*SUMMARY TALK PRESENTED AT THE 9TH INTERNATIONAL WORKSHOP ON DEEP INELASTIC SCATTERING (DIS 2001), BOLOGNA, APRIL 2001. 
- Spin structure functions $g_{1}$ and $g_{2}$

- Polarized parton distributions

- Single-spin azimuthal asymmetry and transversity distribution

- Generalized (skewed) parton distributions and exclusive processes

- Starting experiments and new projects

From the above list of topics, it is quite evident that spin physics is in a rich, exciting and dynamical situation and that a new era, which investigates new types of fundamental distribution functions, is started, while the first era is still far from being exhausted.

After the early measurements performed at SLAC, the modern era in spin physics begun in 1988 when the EMC collaboration reported the experimental evidence that only a small fraction of the proton spin is carried by the quarks $\$$. This shocking result has produced an enormous experimental effort during the 90s when several measurements have been successfully performed at CERN, SLAC and DESY with a precise determination of the spin structure functions. To better underline the importance of the EMC measurement is sufficient to mention that the related publication, together with the old ones on the $J / \psi$ discovery 2 and with the recent one on the atmospheric neutrino oscillationl, is the most cited experimental paper in high energy and nuclear physics of the last 30 years 1 .

From inclusive and semi-inclusive polarized data, the helicity distribution for the up- and down-quarks are now known with reasonably good precision, while still very sparse informations are available for the sea-quark and gluon helicity distributions. These quantities are planned to be measured more precisely in several experiments at DESY, CERN, RHIC and SLACE.

Although the importance of the leading twist transversity distribution $h_{1}(x)$ (often also denoted $\delta q(x)$ ) for completing the knowledge on the spin structure of the nucleon was recognized quite some time agde, only recently the experimental hunt for it started. Unlike the other two leading-twist distribution functions $f_{1}(x)$ and $g_{1}(x)$ (or $q(x)$ and $\Delta q(x)$ ), the trapsversity distribution function is still unknown due to its chiral-odd natured. First HERMES and SMC datal showed that a non zero spin dependent T-odd fragmentation function (the Collins f.f.) allows to access the transversity distribution and even higher-twist helicity changing distributions. More options to unravel transversity - each with its own advantages and caveats due to the subtle interplay of chirality and time-reversal issues - are under current investigation. 
Finally the recently developed formalism of Generalized Parton Distributions (GPDs) for a description of the exclusive leptoproduction reactions has deepened the insight in the connection between exclusive and inclusive hard reactions. Moreover, accessing GPDs experimentally appears to be a promising way to acquire additional and complementary informations on the spin structure of the nucleon 6 . Due to the experimental difficulties to measure exclusive reactions, like Deeply Virtual Compton Scattering (DVCS), at sufficiently hard scale, first results in this field are appearing just now 10 .

\section{Spin structure functions $g_{1}$ and $g_{2}$}

The spin structure function $g_{1}$ is now well determined for proton, deuteron and neutron. Most of the available data cover the DIS region with $Q^{2}>1$ $\mathrm{GeV}^{2}$ and $W>2 \mathrm{GeV}$. Unlike the unpolarized structure functions which have been measured over a broad kinematic range, no polarized data are available for large $Q^{2}>70 \mathrm{GeV}^{2}$ or small $x<10^{-3}$.

In fig. 1 the world data for $g_{1}$ in the DIS region are presented. Together with the already published data, the preliminary HERMES data (see contribution of $C$. Weiskopf) for $g_{1}^{p}$ at low- $x$ and low- $Q^{2}$ and for $g_{1}^{d}$ are also shown. It is worth to note that the latter data set represents only a small fraction $(\sim 1 / 6)$ of the already collected data on the deuteron. An important experimental finding is the $Q^{2}$-independence of the inclusive asymmetry $A_{1} \sim g_{1} / F_{1}$ in the whole measured kinematic range. This suggests a similar behavior of the scaling violation for the structure functions $F_{1}$ and $g_{1}$. After the completion of the analysis of all HERMES data on the deuteron, no substantial improvement of the knowledge of $g_{1}$ in the DIS region of moderate $x$ and $Q^{2}$ is foreseen in the near future.

On the contrary, several measurements are ongoing or are planned in the photoproduction or in the resonance electroproduction regions (see S. Kuhn).

Unlike the unpolarized case, there are no results for the spin-dependent cross section for real photon for which only phenomenological extrapolations from the DIS region are available 11 . First preliminary data collected at MAMI and ELSA have been reported (see K. Helbing) which show the dominance of the helicity $3 / 2$ in the polarized photoabsorption, due to the $P_{33}, D_{13}$ and $F_{15}$ resonance excitation. The GDH collaboration is planning to measure the spin dependent cross sections for the proton and the neutron up to $3 \mathrm{GeV}$ in order to explore the onset of the Regge regime. In addition a new polarized real photon beam facility has been approved at SLAC (see G. Peterson) which will extend this measurement up to $40 \mathrm{GeV}$.

In the nucleon resonance region preliminary results are available from

dis2001 proc: submitted to World Scientific on October 27, 2018 


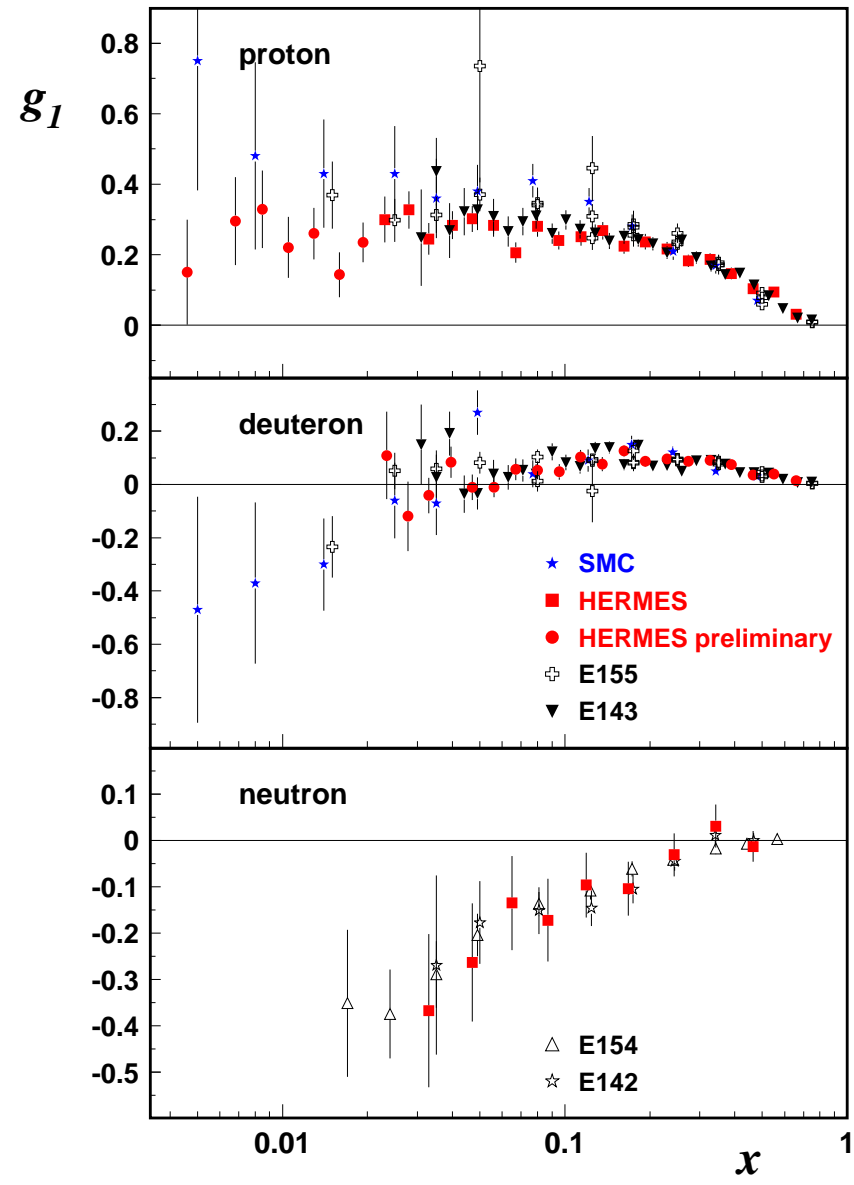

Figure 1. World data on spin structure function $g_{1}$ collected during the 90s. All the data are shown at the measured $Q^{2}$. SMC results for $Q^{2}<1 \mathrm{GeV}^{2}$ at very low $x$ are not shown.

JLab. In fig. 2 the first moment $\Gamma_{1}$ of the spin structure function $g_{1}$ for the proton is shown as measured by the CLAS Collaboration (see G. Dodge). The data show a strong $Q^{2}$-dependence of the integral which becomes negative at very low $Q^{2}$ due to the role of the $P_{33}$ ( $\Delta$-resonance) in this domain. Also reported (see W. Korsch) is the Hall-A Collaboration measurement of generalized GDH integral of the neutron as function of $Q^{2}$ which approach the 


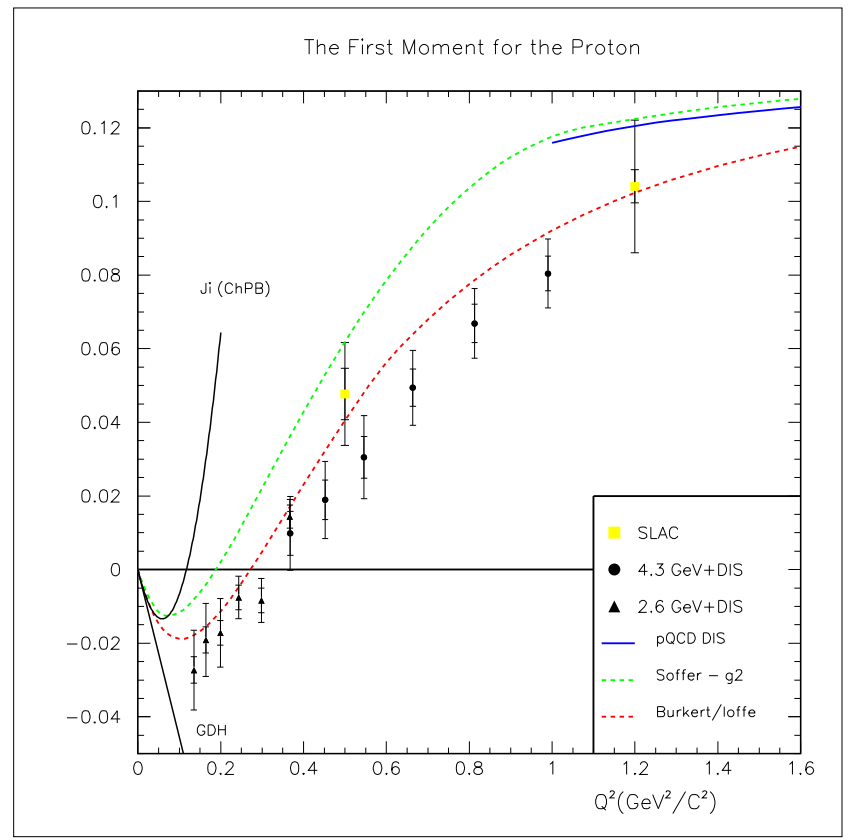

Figure 2. Preliminary results for the first moment of $g_{1}$ for the proton as a function of $Q^{2}$ from CLAS at Jefferson Lab. Also shown are the data from SLAC E143 and different predictions.

Sum Rule expectation value for the real photon. Due to the limited energy of the JLab beam, both $\Gamma_{1}$ and GDH integrals at low $Q^{2}$ include a high energy extrapolation which determination will strongly benefit from the planned real photon measurements.

New and precise results on the spin structure function $g_{2}$ from the SLAC E155x collaboration (see O. Rondon) show for the first time a possible small deviation from the dominant twist-2 contribution (Wandzura-Wilczek term) for the proton. The available SLAC data 12 on $g_{2}$ are shown in fig. 3. These data allow to extract the twist-3 reduced matrix element $d_{2}$ which appears to be slightly positive for both proton and neutron. This result is in contradiction with QCD sum rule models while is in better agreement with quark bag and chiral soliton models. Like for the structure function $g_{1}$ case, no further experimental improvement is foreseen for $g_{2}$ in the DIS regime in the near future. 


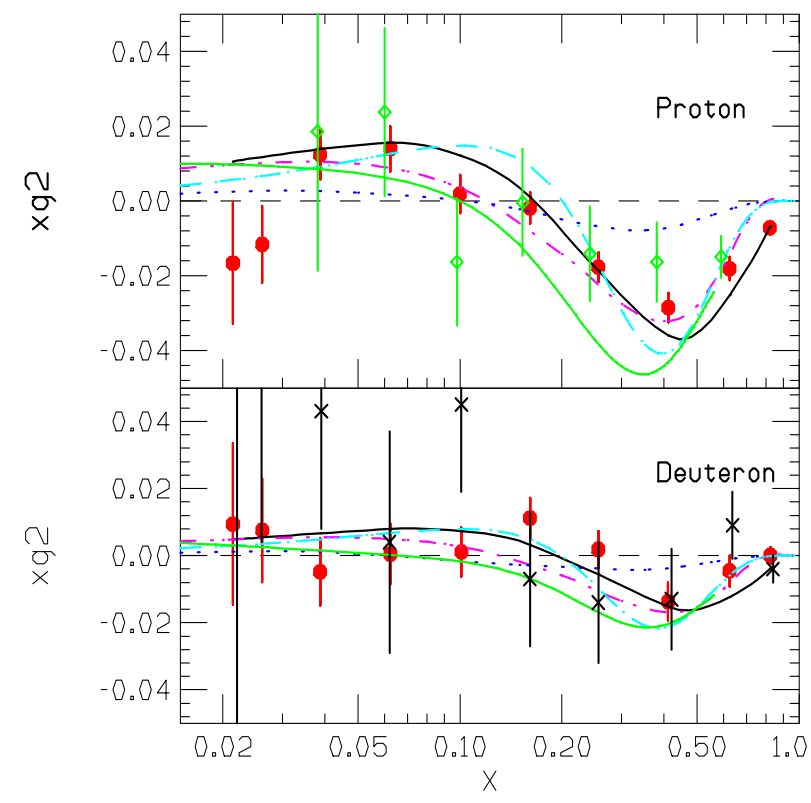

Figure 3. SLAC data on spin structure function $g_{2}$. Close circles are the preliminary E155x results. Curves are predictions from Stratmann, Weigel, Wakamatsu and Song.

\section{Polarized parton distributions}

Inclusive results for spin structure functions allow to extract informations on the quark and the gluon helicity distributions through NLO QCD fits. A 8 parameter fit of $A_{1}$ data, performed in the $\overline{M S}$ scheme and under the $\mathrm{SU}(3)$ assumption, has been presented (see J. Blümlein). In this fit, the parameter correlations were fully taken into account in the Gaussian error propagation Another QCD fit of $A_{1} \sim g_{1} / F_{1}$ data in the $J E T$ scheme has been showed 13 (see D. Stamenov). The advantage of fitting $A_{1}$ instead of $g_{1}$ results in the cancellation of higher twist effects for $Q^{2}$ down to $1 \mathrm{GeV}^{2}$. These new fits confirmed that inclusive data alone poorly constrain the separate quark and anti-quark polarized distributions and the gluon distribution as well (see $A$. Miller).

Combined inclusive and semi-inclusive QCD analysis have been performed 14 and showed that semi-inclusive data 15 are in perfect agreement with the inclusive data with respect to the determination of polarized par- 


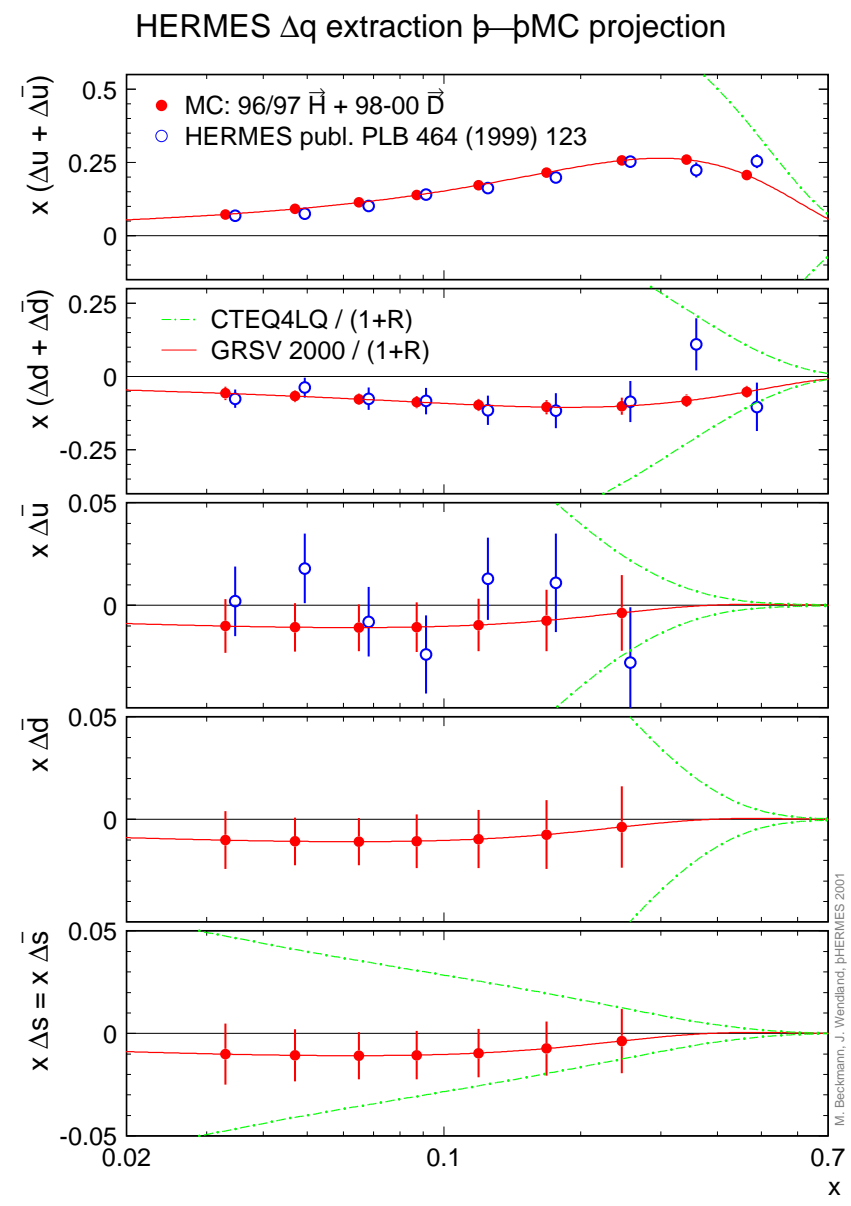

Figure 4. Expected precision for polarized quark distributions from the inclusive and semiinclusive data already collected by HERMES and with no flavor symmetry assumption on the sea quarks. Also shown are the published HERMES results based on 1995-1997 runs.

ton densities, but constitute a very powerful tool to study the sea quark polarization $(\Delta \bar{u}, \Delta \bar{d}$ and $\Delta s)$. An updated analysis of the HERMES results with the inclusion of the preliminary deuteron data has been shown (see $M$. Beckmann). This analysis, based on the purity formalism, is still based on a symmetric assumption of the sea. Since the unpolarized parton densities of 
the sea quarks are not flavor symmetric, there is no reason to believe that this symmetry should hold for the polarized case. Indeed there is a suggestion based on a meson-cloud model that $\Delta \bar{u}-\Delta \bar{d}<0$ (see M. Miyama). The full analysis of HERMES data with the inclusion of the large statistics on the deuteron and the particle identification with the RICH detector will allow not only to strongly improve the statistical precision on the polarization of the upand down-flavors but in addition to determine the polarization for all different sea flavors without symmetry assumption (see fig. (4). The polarized quark distributions will be also studied in the near future at COMPASS with semiinclusive hadron leptoproduction and at RHIC through the parity violating processes $u \bar{d} \mapsto W^{+} \mapsto l^{+}+\nu$ and $d \bar{u} \mapsto W^{-} \mapsto l^{-}+\nu$.

One of the most important issues in spin physics for the next 5 years will be the measurement of the gluon polarization which is poorly determined by NLO QCD fits. Gluon polarization will be studied at COMPASS, SLAC and RHIC by measuring the open charm production and the high- $p_{T}$ hadron pairs in the photon-gluon fusion in $\vec{\gamma}+\vec{p}$ experiments and in the direct $\gamma$ production and two-jet events in $\vec{p}+\vec{p}$ experiments. In addition running RHIC at very high energy $(\sqrt{s}=500 \mathrm{GeV})$ will allow to access the gluon polarization through the heavy quarks (charm and bottom) hadroproduction (see I. Bojak). In fig. 5 the projected errors of the planned measurements are shown together with the first HERMES data 16 which interpretation is improving by the better knowledge of all the competing sub-processes (see E.C. Aschenauer).

COMPASS will start data taking this year using the previous SMC polarized target (see F.H. Heinsius). Also the RHIC spin physics program will start gradually this year and the full luminosity and the complete of the EM calorimeter of the STAR detector will be reached in 2004 (see L. Bland). SLAC experiment E161 is planned to run in 2003-2004 (see G. Peterson).

There is a number of proposals for the medium-range future, where high luminosity experiments will strive for unprecedented precision in the determination of polarized distribution functions and the gluon polarization as some of their main goals: the TESLA-N experiment, or experiments at the ELFE accelerator (see D. Ryckbosch), as well as experiments at the proposed EIC accelerator (see A. Desphande) into which the former eRHIC and EPIC proposals have merged.

\section{Single spin azimuthal asymmetries: Towards a global analysis of transversity}

Only the knowledge of the transversity distribution $h_{1}(x)$ will complete the mapping of the spin structure of the nucleon at leading twist. Due to its 


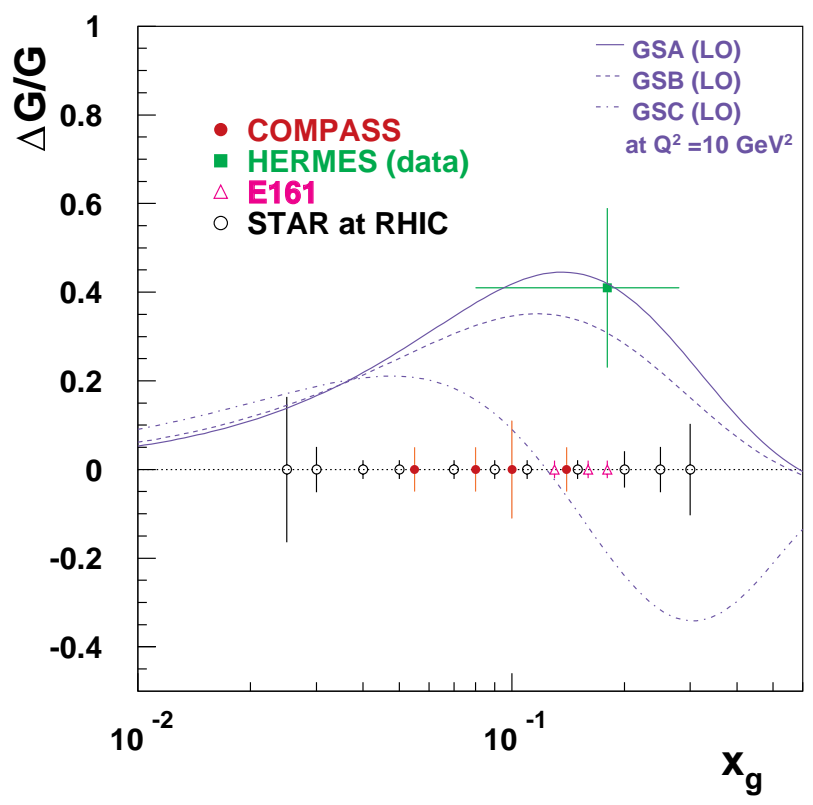

Figure 5. Expectations for future measurements of the gluon polarization. The HERMES result for high- $p_{T}$ hadron pairs is also shown. The curves are QCD analysis of polarized inclusive structure function.

chiral-odd nature transversity is inaccessible in totally inclusive DIS; in order to form a chiral-even observable it requires the presence of a second chiralodd projection of a soft matrix element in a more complex reaction - a second transversity distribution, a higher twist distribution, or an appropriate fragmentation function 17 . The object of desire will consequently appear in an observable together with a second yet unknown function, usually in form of a convolution. Therefore, complementary experiments have to be utilized in a combined analysis in order to finally extract the transversity distribution.

This situation not only represents a considerable high level of sophistication on the theoretical side, but also causes tremendous demands on experimental setups. Thus, only now we are in the situation to seriously tackle unraveling of transversity.

At this workshop to our knowledge truly the complete set of presently known ways to access transversity has been discussed in one or the other talk. The most promising approaches are sketched below. 


\subsection{Collins effect in leptoproduction; a Single Spin Asymmetry}

An azimuthal asymmetry induced by the so-called Collins effect 18 is probably the most prominent representative of the different observables involving the transversity distribution. Its variant in 1-hadron inclusive lepton-nucleon scattering predicts a characteristic angular dependence in the azimuthal distribution of detected pions in the final state. The chain of logic is as follows: The transversity distribution describes how much of a transverse target polarization is transferred to a transversely polarized quark; the Collins fragmentation function, dubbed $H_{1}^{\perp}\left(z, k_{T}\right)$ in the Amsterdam naming scheme 19 , describes the transfer of transverse quark polarization into preferred directions in the azimuthal distribution of produced hadrons, say pions.

The observation of this asymmetry has been reported by HERMES and SMCE. In fig. 6 the HERMES data are shown. Though the target polarization longitudinal to the lepton beam on the average corresponds to only small components of polarization transverse to the photon direction, the $\sin (\phi)$ dependence is rather convincingly confirmed for positively charged and neutral pions (see contribution of D. Hasch). A comparison with theoretical estimates and limits shows good agreement (see K. Oganessyan).

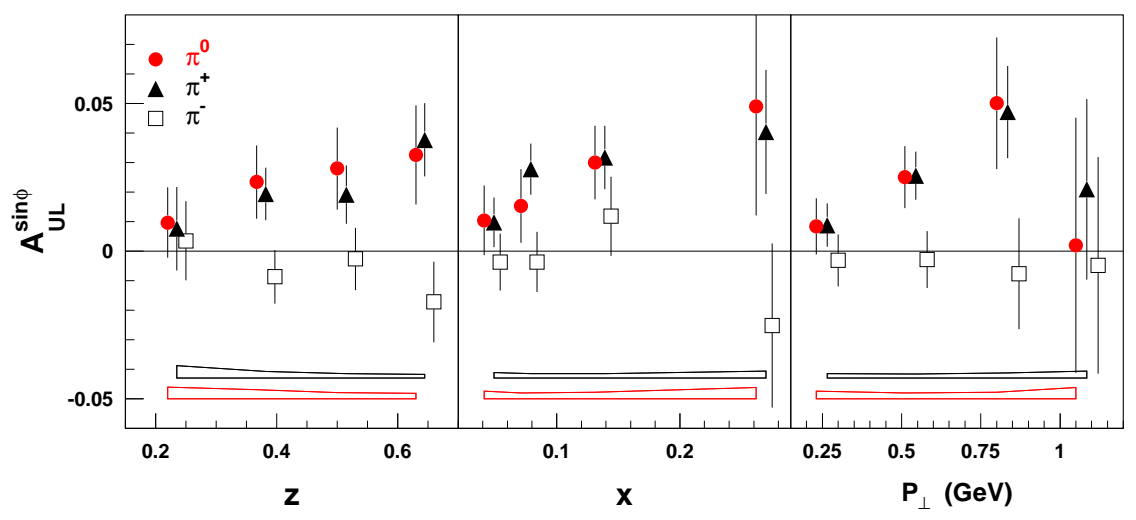

Figure 6. Single spin azimuthal asymmetry in electroproduction of pions in deep inelastic scattering (Collins effect) measured at HERMES. Shown is the $\sin (\phi)$ moment $A_{U L}^{\sin }$ for unpolarized beam and longitudinally polarized target vs. $z, x$, and $p_{T}$ (see D. Hasch).

The effect of higher order perturbative corrections have to be taken into account in an analysis of the asymmetries. For observables, which involve two scales like the Collins effect, there is a non-collinear factorization and 
the cancellation of double-logarithms is incomplete. The remaining soft gluon corrections sum up to Sudakov suppression factors. These Sudakov factors have been estimated to have significant effects on the magnitude of observed asymmetries 20 (see D. Boer). There is also very recent progress on the understanding of the evolution of the Collins fragmentation function, a question which is complicated by the fact that dependence on transverse momentum is to be kept. An analysis on this issue, and the resulting evolution equation for $H_{1}^{\perp}$ in the large- $N_{C}$ limit was presented21 (see P. Mulders). Both reported results constitute important progress towards a complete treatment of azimuthal asymmetries beyond the leading order.

\subsection{Transversely polarized $\Lambda$ 's in a Double Spin Asymmetry}

Another opportunity to access transversity is to measure the transverse polarization of produced $\Lambda$ 's in semi-inclusive DIS. The $\Lambda$ 's are self-analyzing, i.e. they reveal their spin by the subsequent decay into nucleon and pion, whose momenta have to be determined.

The use of this possibility, which will become important with the improved particle identification of future and starting experiments, relies on the knowledge of competing effects which also may result in transverse polarization of $\Lambda$ 's. For instance, also unpolarized quarks can induce a transverse polarization of $\Lambda$ 's depending on their azimuthal distribution in the jet22. This effect involves the only other T-odd leading-twist fragmentation function $D_{1 T}^{\perp}\left(z, k_{T}\right)$, quasi the 'mirror image' of the Collins f.f. (see F. Murgia).

Also a better understanding of the longitudinal spin transfer from quarks to $\Lambda$ 's may shed light on this difficult issue (see O. Grebenyuk for experimental and U. D'Alesio for theoretical aspects).

\subsection{Double Spin Asymmetries in semi-inclusive $\rho$ production}

Produced $\rho$ 's in semi-inclusive DIS also reveal their spin via the distributions of the decay products, i.e. pion pairs. It turns out that in principle there are three azimuthal asymmetries involving the transversity distribution together with different spin-1 fragmentation function $\$ 23$ (see A. Bacchetta). The involved fragmentation functions can be extracted from the mass spectrum of pion couples; thus, they have to be in close relationship with the two-pion f.f. discussed in the following paragraph. 


\subsection{Single Spin Asymmetry in $\pi \pi$ production; interference fragmentation functions}

A very promising option to access transversity is given by a Single Spin Asymmetry in the production of pion pairs in the same current jet2 3 . The relative momentum within the pair provides an additional lever arm to extract the desired information. This allows for the integration over transverse momentum of the pion pair relative to the jet axis, which leads to a collinear factorization and the vanishing of Sudakov suppression factors (see D. Boer). The necessary T-odd and chiral-odd fragmentation functions are induced by interference effects between two different channels for the production of the pion couple in the hadronization of the jet. The resulting azimuthal asymmetry in two-pion inclusive DIS was discussed, and a model estimate for the involved interference fragmentation function reported (see M. Radici).

\subsection{Transversity perspectives}

The aforementioned options are currently under active theoretical investigation. First experimental observations of transversity related azimuthal asymmetries are reported, and a good share of future experimental efforts, for instance at HERMES and COMPASS experiments, and part of the RHIC spin program, will go into the 'transversity project'. Experimental feasibility studies and projections appear to be very promising for the near future.

For the extraction of the transversity distribution the analyses of the asymmetries will have to be supplemented by independent knowledge on some fragmentation functions, for which $e^{+} e^{-}$data (for instance from LEP experiments, or data from the future $B$-factories) seem to be the best possible source of information.

Most likely, there will not be one golden way to the extraction of the transversity distribution, but the need for a 'global transversity analysis' (D. Boer). In fact, the enterprise has already started.

\subsection{Remark}

Sometimes the, well justified, excitement about the transversity distribution as the missing spin distribution function obscures the clearness of the general aim: to collect as much as possible information and insight into the mechanism of confinement, and thus into the inner structure of hadrons. The knowledge on complicated spin dependent fragmentation functions, and for instance higher-twist distribution functions (see e.g. Y. Koike for a theoretical approach to twist-three functions from gluonic pole 25 ), is not merely a 
prerequisite for a transversity analysis . The effort on extracting those functions is justified in its own interest as steps towards a full understanding of confinement at work.

\section{Generalized parton distribution and exclusive processes}

\subsection{Why should we bother to measure GPDs?}

In fact, this is not the right question to be asked, since it is practically impossible to avoid dealing with GPDs when utilizing hard reactions to unravel the inner structure of hadrons. Every form factor is an $x$-moment of a GPD; every ordinary distribution function is given by a GPD in a certain kinematical limit. From this point of view GPDs have been studied at least for 50 years. A more sensible question would be: What new aspects can we learn from GPDs, inaccessible with ordinary parton distributions and form factors?

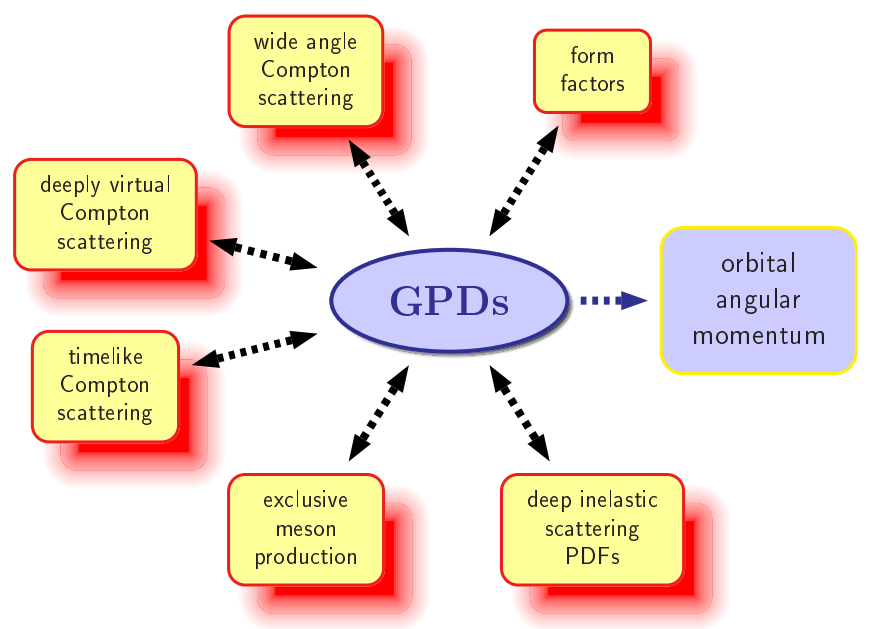

Figure 7. Generalized parton distributions occur in the description of many different hard reactions; sometimes in the form of $x$-moments or in special kinematical limits.

The great advantage of the concept of GPDs certainly is a unifying framework for exclusive and inclusive reactions. The GPDs have a sound theoretical basis, their definition in QCD as Fourier transforms of hadronic matrix elements is a direct generalization of the one for ordinary PDFs, their evolution is known, the relationship to form factors, parton densities is well- 
established262728. For the kerreactions involving GPDs factorization theorems have been worked out 29,28. Justifiably, these elements of the formalism can already be considered 'future textbook knowledge' on hard reactions.

A representation of GPDs as overlaps of light-cone wave functions allows a clear interpretation 30 . Whereas ordinary PDFs are parton densities, i.e. probabilities, the GPDs constitute interference amplitudes between different kinematical situations. The information carried by GPDs thus exceeds the one of PDFs, just as there is more to be learned from an amplitude then from its absolute square. Not only longitudinal degrees of freedom, but also transverse d.o.f. are accessible; GPDs know about the orbital angular momentum of partons inside hadrons, which is obvious from the hadron-helicity changing matrix elements and the corresponding distribution functions, which are not present in the forward case of ordinary PDFs. Moreover, the $q \bar{q}$ components of the nucleon can be directly studied from a kinematical domain again not accessible in forward reactions (for more details see the contribution by M. Diehl).

\subsection{Observability of hard exclusive reactions in the deeply virtual domain}

Surely, there is a price to pay for the rich information contained in GPDs. These are objects depending on three variables (and have an additional logarithmic scale dependence). Two variables are controlled by external momenta, the momentum transfer squared $-t$, and the analogue of the Bjorken variable $\xi$. The third variable $x$ constitutes an internal d.o.f. of the process and is integrated over, which presents an additional challenge, since observables will have to be deconvoluted to access the GPDs.

In addition, hard exclusive reactions involving GPDs are difficult to measure, since counting rates typically drop down drastically with increase of the hardness of the process. Nevertheless, there is great progress on the experimental side. Several observations have been reported on the key processes to GPDs, like the DVCS signal from ZEUS and the DVCS cross section measurement at $\mathrm{H} 1$ (see fig. 8 and fig. 9 and the contribution by L. Favart)

Even azimuthal asymmetries in the DVCS process have been already reported. In fig. 10 the beam-spin asymmetry observed by HERMES is displayed, together with a $\sin (\phi)$-moment of the cross-section showing a clear non-vanishing effect in dependence on the missing mass. Though the final state could not be identified, the value of the asymmetry close to the proton mass can be assigned mainly to the Compton process. This result is very promising in view of improved particle identification available soon at HERMES (see J. Volmer). 


\section{ZEUS 1996/97 Preliminary}
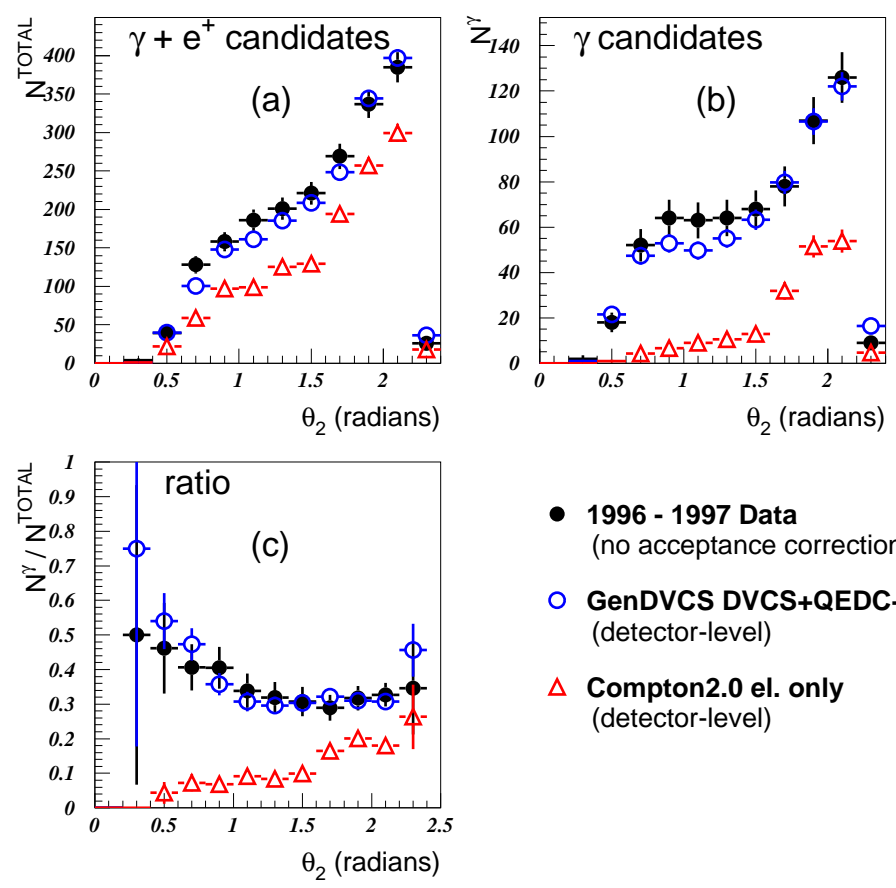

- 1996 - 1997 Data (no acceptance correction)

O GenDVCS DVCS+QEDC+int. (detector-level)

$\Delta$ Compton2.0 el. only (detector-level)

Figure 8. Observation of the DVCS reaction $e^{+} p \rightarrow e^{+} \gamma p$ at HERA in data taken with the ZEUS detector (see L. Favart).

Similarly at HERMES an azimuthal asymmetry in hard exclusive leptoproduction of $\pi^{+}$has been observed. In fig. 11 the asymmetry is shown together with the $\sin (\phi)$-moment vs. $x$ (see E. Thomas).

Only about three years ago, it was general believe that exclusive reactions in the deeply virtual kinematical domain were forbiddingly difficult to measure. All the above mentioned findings prove the feasibility of those measurements. The more can be expected from dedicated experiments or even dedicated accelerators like ELFE (see contribution by D. Ryckbosch) or EIC (see A. Desphande) tuned to the special needs of the observation of hard exclusive processes. 

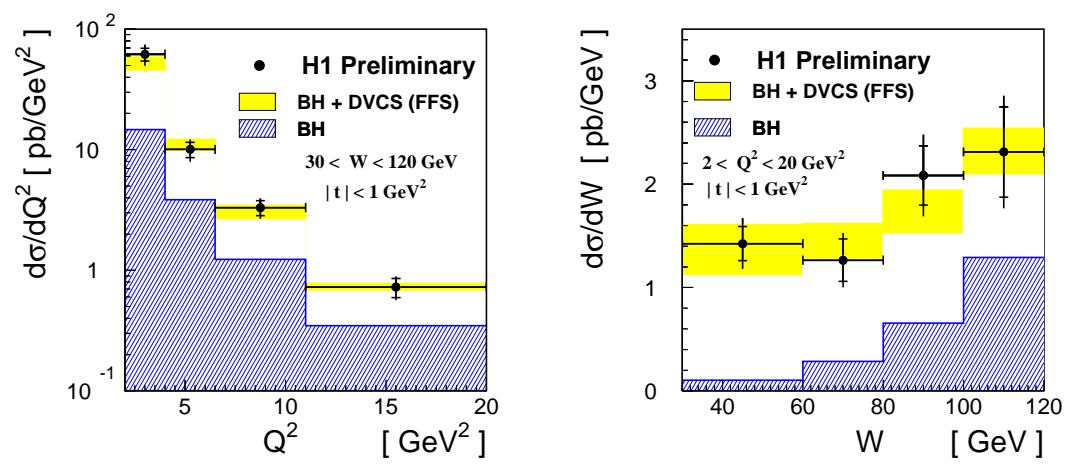

Figure 9. Differential cross section measurements for the DVCS reaction $e^{+} p \rightarrow e^{+} \gamma p$ from $\mathrm{H} 1$ as a function of $Q^{2}$ (left) and $W$ (right). The hatched histogram shows the contribution of the Bethe-Heitler process to the reaction. (see L. Favart)
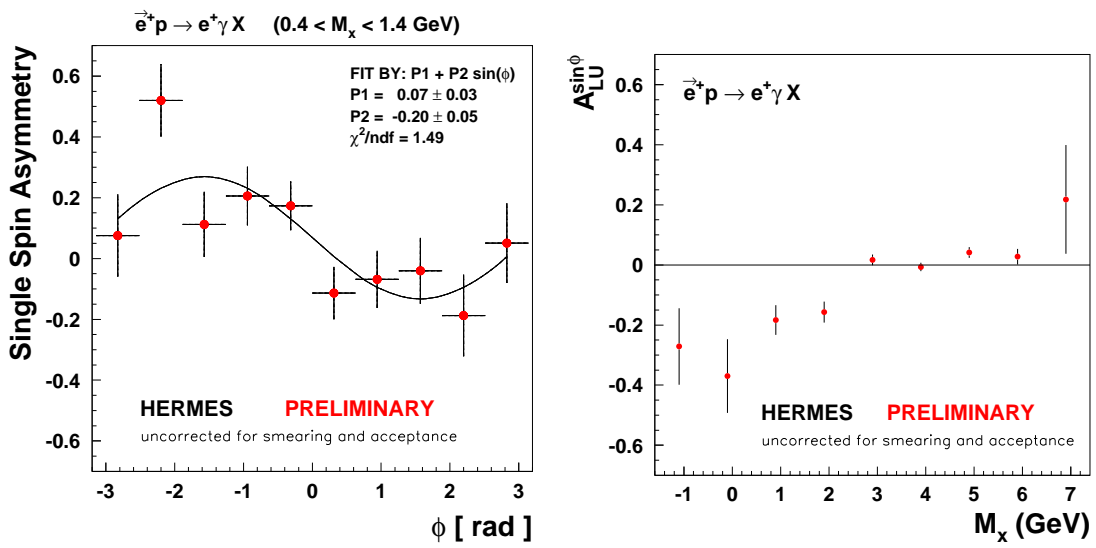

Figure 10. The DVCS Single Spin Asymmetry from HERMES. Left: Beam-spin asymmetry $A_{L U}$ for hard electroproduction of photons as a function of the azimuthal angle $\phi$. The data correspond to the missing mass region between 0.4 and $1.4 \mathrm{GeV}$. Right: The beam-spin analyzing power $A_{L U}^{\sin }$ for hard electroproduction of photons on hydrogen as a function of the missing mass (see J. Volmer).

\subsection{Some aspects of the theoretical status of GPDs}

Likewise, on the theoretical side there has been great progress. It has been shown that charge, spin and azimuthal asymmetries with longitudinal and 

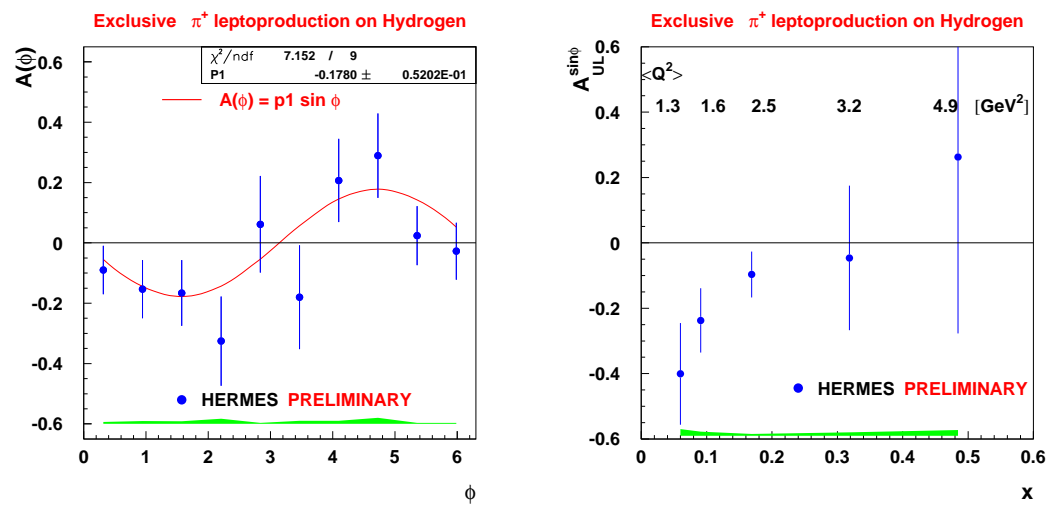

Figure 11. Azimuthal asymmetry in exclusive $\pi^{+}$leptoproduction. Left: The asymmetry vs. $\phi$. Right: The $\sin (\phi)$ moment of the polarized cross section vs. $x$ (see E. Thomas).

transversely polarized targets allow to disentangle all twist-2 and twist-3 GPDs when use is made of the interference with the Bethe-Heitler proces: 31 (see D. Müller). NLO $\alpha_{S}$ corrections and evolution of GPDs from two-loop diagrams have been calculated 32 . From the results the interesting physical pictures arises that contrary to expectation meson-like excitations still contribute at small $x$-values, and at large $x$-values the sea-quark contributions are non-negligible, which seems to indicate that even in the valence region one does not necessarily strike the valence quarks directly (see A. Freund).

Probably the strongest point in the theoretical concept of GPDs is the fact that they link so many different processes. Key reactions are certainly the DVCS and hard electroproduction of mesons. But also large momentum transfer reactions are sensitive to GPDs, where they contribute through $\xi=0$ moments. The information obtainable from large momentum transfer exclusive processes is complementary to the one from deeply virtual reactions. Newly defined Compton form factors reflect the large $t$ dependence of the GPDs, similarly as elastic form factors do. With models for light-cone wave functions one can achieve not only good agreement with elastic form factor and Real Compton Scattering (RCS) data, but also arrive at interesting predictions for polarized and unpolarized RCS, VCS and electroproduction of mesons at large momentum transfer 33 (see P. Kroll).

Another option to access GPDs is the inverse DVCS, or timelike CS, process $\gamma p \rightarrow \mu^{+} \mu^{-} p$ with a large invariant mass of the lepton pair. It was 
shown that the process can be described in a factorized form similar to DVCS, and numerical estimates for its cross section have been given (see M. Diehl).

The most intriguing aspect in the formalism is the information carried by the GPDs on orbital angular momentum. Actually, Ji's proposal27 on a gauge invariant decomposition of the nucleon spin initiated the renewed great interest in deeply virtual exclusive reactions. There is some theoretical debate on the issue: it appears that the elements in one possible decomposition of the nucleon spin can be measured, but have no direct parton representation, or in a different decomposition, the terms are separately interaction independent, gauge invariant integrals over parton densities, but there is no known way to measure them 34 .

Anyway, there is general agreement that the information on orbital angular momentum of partons is carried by GPDs; the situation is a challenge to theory to find the appropriate way to isolate the desired information. A contribution to the ongoing debate is the development of a physical picture of the transverse localization of orbital angular momentum. Let $\Delta_{T}$ be the transverse part of the momentum transfer in the process. The measurement of the $\Delta_{T}$ dependence of amplitudes can be inverted by Fourier transform to find the spatial impact parameter $b_{T}$ location of the partons. The transverse structure is directly observable when amplitudes are measured by interference (see J. Ralston).

\subsection{GPD perspectives}

The exploration of the concept of Generalized Parton Distributions has just begun. The theoretical sound basis has been carried to the NLO both in $\alpha_{s}$ corrections and evolution, and in higher-twist corrections. First experimental observations on DVCS cross sections, asymmetries and hard exclusive mesonproduction have confirmed the feasibility of measurements.

Spin physics will serve as the most important tool to access the different GPDs in a variety of asymmetries in many different reactions. The interplay between spin degrees of freedom and orbital angular momentum of partons in building up the nucleon spin will be the key to understand the inner structure of hadrons.

\section{Conclusion}

The physics goals in the field of hadron physics are clearly defined. Experiments have already successfully provided a great deal of information on the nucleon spin structure, and have proven to be capable to explore the still

dis2001 proc: submitted to World Scientific on October 27, 2018 
missing elements and new theoretical concepts. We are looking forward to an exciting future.

\section{Acknowledgments}

This work has been supported by the TMR network HPRN-CT-2000-00130.

\section{References}

1. J. Ashman et al. [European Muon Collaboration], Phys. Lett. B 206 (1988) 364.

2. J. J. Aubert et al., Phys. Rev. Lett. 33 (1974) 1404;

J. E. Augustin et al., Phys. Rev. Lett. 33 (1974) 1406.

3. Y. Fukuda et al. [Super-Kamiokande Collaboration], Phys. Rev. Lett. 81 (1998) 1562.

4. SPIRES TOPCITE 1000+ Database.

5. For a recent review see B. W. Filippone and X. Ji, hep-ph/0101224.

6. J. P. Ralston and D. E. Soper, Nucl. Phys. B 152 (1979) 109;

R. L. Jaffe, hep-ph/9602236.

7. For a recent review see V. Barone, A. Drago and P. G. Ratcliffe, hep-ph/0104283.

8. A. Airapetian et al. [HERMES Collaboration], Phys. Rev. Lett. 84 (2000) 4047; A. Airapetian et al. [HERMES Collaboration], hepex/0104005; A. Bravar [Spin Muon Collaboration], Nucl. Phys. Proc. Suppl. 79 (1999) 520.

9. For a recent review see K. Goeke, M. V. Polyakov and M. Vanderhaeghen, hep-ph/0106012.

10. A. Airapetian et al. [HERMES Collaboration], hep-ex/0106068;

C. Adloff et al. [H1 Collaboration], hep-ex/0107005;

P. R. Saull et al. [ZEUS Collaboration], hep-ex/0003030;

S. Stepanyan et al. [CLAS Collaboration], hep-ex/0107043.

11. N. Bianchi and E. Thomas, Phys. Lett. B 450 (1999) 439.

12. K. Abe et al. [E143 Collaboration], Phys. Rev. Lett. 76 (1996) 587;

P. L. Anthony et al. [E155 Collaboration], Phys. Lett. B 458 (1999) 529.

13. E. Leader, A. V. Sidorov and D. B. Stamenov, Phys. Rev. D 58 (1998) 114028.

14. D. de Florian and R. Sassot, Phys. Rev. D 62 (2000) 094025.

15. B. Adeva et al. [Spin Muon Collaboration], Phys. Lett. B 420 (1998) 180; K. Ackerstaff et al. [HERMES Collaboration], Phys. Lett. B 464 (1999) 123. 
16. A. Airapetian et al. [HERMES Collaboration], Phys. Rev. Lett. 84 (2000) 2584.

17. R. L. Jaffe, hep-ph/9710465.

18. J. Collins, Nucl. Phys. B 396 (1993) 161.

19. P. J. Mulders and R. D. Tangerman, Nucl. Phys. B 461 (1996) 197 [Erratum-ibid. B 484 (1996) 538];

D. Boer, R. Jakob and P. J. Mulders, Nucl. Phys. B 564 (2000) 471.

20. D. Boer, Nucl. Phys. B 603 (2001) 195.

21. A. A. Henneman, D. Boer and P. J. Mulders, hep-ph/0104271.

22. M. Anselmino, D. Boer, U. D'Alesio and F. Murgia, Phys. Rev. D 63 (2001) 054029.

23. A. Bacchetta and P. J. Mulders, Phys. Rev. D 62 (2000) 114004.

24. J. C. Collins, S. F. Heppelmann and G. A. Ladinsky, Nucl. Phys. B 420 (1994) 565; J. C. Collins and G. A. Ladinsky, hep-ph/9411444; X. Artru and J. Collins, Z. Phys. C 69 (1996) 277; R. L. Jaffe, X. Jin and J. Tang, Phys. Rev. Lett. 80 (1998) 1166; A. Bianconi, S. Boffi, R. Jakob and M. Radici, Phys. Rev. D 62 (2000) 034008.

25. Y. Kanazawa and Y. Koike, Phys. Rev. D 64 (2001) 034019.

26. D. Muller, D. Robaschik, B. Geyer, F. M. Dittes and J. Horejsi, Fortsch. Phys. 42 (1994) 101.

27. X. Ji, Phys. Rev. Lett. 78 (1997) 610.

28. A. V. Radyushkin, Phys. Rev. D 56 (1997) 5524.

29. J. C. Collins, L. Frankfurt and M. Strikman, Phys. Rev. D 56 (1997) 2982; X. Ji and J. Osborne, Phys. Rev. D 58 (1998) 094018; J. C. Collins and A. Freund, Phys. Rev. D 59 (1999) 074009.

30. M. Diehl, T. Feldmann, R. Jakob and P. Kroll, Nucl. Phys. B 596 (2001) 33 [Erratum-ibid. B 605 (2001) 647].

31. M. Diehl, T. Gousset, B. Pire and J. P. Ralston, Phys. Lett. B 411 (1997) 193; A. V. Belitsky, D. Muller, L. Niedermeier and A. Schafer, Nucl. Phys. B 593 (2001) 289; A. V. Belitsky and D. Muller, Nucl. Phys. B 589 (2000) 611; A. V. Belitsky, A. Kirchner, D. Muller and A. Schafer, Phys. Lett. B 510 (2001) 117.

32. A. Freund and M. F. McDermott, hep-ph/0106319.

33. M. Diehl, T. Feldmann, R. Jakob and P. Kroll, Eur. Phys. J. C 8 (1999) 409; M. Diehl, T. Feldmann, R. Jakob and P. Kroll, Phys. Lett. B 460 (1999) 204; H. W. Huang and P. Kroll, Eur. Phys. J. C 17 (2000) 423.

34. R. L. Jaffe, Phil. Trans. Roy. Soc. Lond. A 359 (2001) 391. 